\title{
The polarization approach to registration femtosecond time slots based stimulated photon echo on the exciton states
}

\author{
I.I. Popov ${ }^{1, *}$, N.S. Vashurin ${ }^{1}$, S.E. Putilin ${ }^{2}$, E.A. Vinogradov ${ }^{3}$, and A.U. Bahodur ${ }^{1}$ \\ ${ }^{1}$ Volga State University of Technology, 424000 Yoshkar-Ola, Russia \\ ${ }^{2}$ Information Technologies, Mechanics and Optics University, 197101 Saint-Petersburg, Russia \\ ${ }^{3}$ Institute for Spectroscopy RAS, 108840 Troitsk, Moscow, Russia
}

\begin{abstract}
It is reported about the first experiments on the registration of a femtosecond time interval using the effect of non-Faraday rotation of the plane polarization of the stimulated photon echo. The experiment was performed at room temperature in a three-layer semiconductor film thickness of $300 \mathrm{~nm}$, obtained by magnetron sputtering. The fundamental difference of this effect from the Faraday's effect was shown. We give conclusions about the possibility of femtosecond time intervals registration at room temperature with an accuracy of $25 \mathrm{fs}$ in the polarization principle of action, rather than spectral.
\end{abstract}

The aim of the work is aimed at demonstrating the possibility of building on the basis of the effect of the new femtosecond magneto-optics principle of femtosecond time intervals registration. This principle of operation is used polarizing approach to fixing the value of these slotsTo achieve this goal have been made the work for the detection [1] and for quantitative assessment of non-Faraday rotation of the polarization plane of femtosecond stimulated photon echo. This femtosecond stimulated photon echo was observed at room temperature on exciton states of thin textured semiconductor film.

The research method is based on the polarization properties of a stimulated photon echo. Resonance medium - is textured three-layer semiconductor film $\mathrm{ZnO} / \mathrm{Si}(\mathrm{B}) / \mathrm{Si}(\mathrm{P})$ (each layer having a thickness of $100 \mathrm{~nm}$ ) obtained by magnetron sputtering. The film consists of crystalline fibers oriented orthogonally to the film surface. The distance between the fibers is filled with X-ray amorphous phase sprayed substances. Crystal fiber with distance from the substrate decreased the transverse dimensions. This led to the emergence surface nanoscale crystal defects. Open (deformation) of the crystal structure of the fibers at the border of contact with the X-ray amorphous phasecreates conditions for the localization of excitons on these surface defects and creates conditions of inhomogeneous broadening of the excitonic spectral lines.

In theory, this effect is predicted for the gas atmospheres in the work [3] The first experiment on non-Faraday rotation of the plane polarization of the stimulated photon echo made by the authors of [4] Details polarization dependence of this effect is studied in [5] For the first time this effect on exciton gas detected experimentally in $[1,2]$. The proposed polarization approach to the registration ultrashort time intervals of at least an order of magnitude superior to existing methods based on the spectrum approach, using devices

\footnotetext{
* Corresponding author: popov@volgatech.net
} 
such as smiling in the "Pikohron" with a resolution of up to $0.5 \mathrm{ps}$. Discussed in this paper, the effect, in contrast to the rotation of the polarization plane in optically active (chiral) media and the Faraday rotation of the plane of polarization does not depend on the concentration of the growth of the active particles and the length of the path traversed by the radiation in resonance mediumAt the same time, the angle of rotation of the polarization plane of the echo signal $\varphi$ defines by type of quantum transition and magnetic field produced by the amplitude and time delay between the exciting laser pulses. Conducted and the rank of the angle $\varphi$ exceeds the value of the Faraday rotation by 3-4 orders of magnitude $\left(10^{3}-10^{4}\right)$. The authors of [4] it is shown that, depending on the type of transition, degenerate in the magnetic quantum number, $(\mathrm{J} \leftarrow$ for molecules transitions (this corresponds to the spectral lines P -, R or Q-type) for line P -, R-type, this rotation is of the order of degrees, and for Q-type lines, respectively, 90 degrees. This corresponds to the theoretical predictions of this effect .

One of the prerequisites for the emergence of the non-Faraday effect rotation of the plane of polarization of the photon echo is different splitting in a magnetic field of the upper and lower levels of the quantum transition [6]. If we neglect the influence of the exciton-phonon interaction on the formation of the echo signal, taking into account this error occurs when using the analytical expressions for gas at a fixed value of the longitudinal magnetic field component in our experiments, 0.4 Tesla, we can estimate the degree of dependence of the angle SPE polarization plane rotation $\varphi$ of the time interval between the second and third exciting pulses. This relationship will have a form of the function $\varphi=f\left(\operatorname{arctg} \tau_{23}\right)$. To exclude a contribution to the turnaround time interval $\tau_{12}$, it is assumed to be zero. Figure 1 shows the discovery non-Faraday SPE rotation of the plane of polarization in the thin-layer film and its relationship to the magnitude of the time interval between the second and third laser pulses, the excitation signal photon echo.

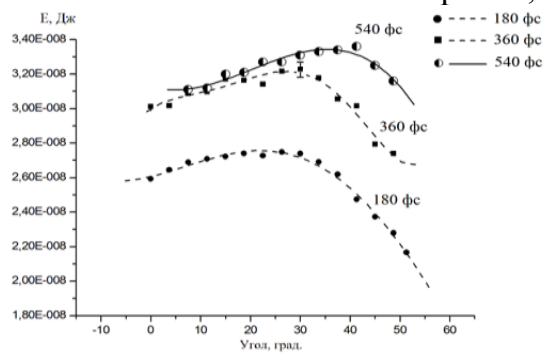

Fig. 1. Dependence non-Faraday rotation of the plane of polarization of the stimulated photon echo on the value of the time interval between the second and the third exciting pulses: at $\tau 23180 \mathrm{fs}$, the rotation angle was 25 degrees, with $360 \mathrm{fs}-32$ degrees, at $540 \mathrm{fs}$ -40 degrees.

From Fig. 1, one can see that registration by changing the angle $\varphi$, consisting of 7 or 8 degrees allows to determine the time interval $\tau_{23}$, amounted to $180 \mathrm{fs}$. Using non-Faraday rotation of the plane of polarization of the stimulated photon echo one can determine the magnitude of the time intervals of 180 fs to 25 fs accuracy of registration degree.

Unusual behavior of the dysprosium CF energies in $\mathrm{Dy}_{2} \mathrm{BaNiO}$ was detected in the temperature range lower than $T_{N}$. All CF levels experience a shift (up to $10 \mathrm{~cm}^{-1}$ ) from their positions at $\mathrm{T}_{\mathrm{N}}$ (see, e.g., Fig. 1). Such behavior can be explained as being due to the spontaneous electric polarization that appears in the temperature range discussed [8]. Redistribution of the charge density influences the crystal field acting on dysprosium and leads to a renormalization of CF self-energies.

\section{References}

1. I.I. Popov et al., Proc. of $10^{\text {th }}$ Int. Sci. Sch. "Sci. \& Innovations" (Yoshkar-Ola, 2015)

2. I.I. Popov, Memoirs of the Faculty of Physics Moscow St. Univ. 4, 154338 (2015)

3. I.V. Evseev, V.M. Ermachenko, V.A Rechetov, Opt. Spectrosc. 52, 444 (1982)

4. I.S. Bikbov, I.I. Popov, V.V. Samartsev, I.V. Yevseyev, Las. Phys. 5, 580 (1995)

5. I.V. Yevseyev et al., Las. Phys. 16, 535 (2006)

6. A.I. Alekseev, JETP Lett. 9, 472 (1969) 